\title{
Doit-on changer rapidement le paradigme de la prise en charge des oesophages de Barrett?
}

\author{
Do we have to move swiftly to change the paradigm in the management of Barrett's oesophagus?
}

\section{Heresbach}

(C) Springer-Verlag France 2011

Ce sujet est à lui seul la synthèse et la caricature des systèmes et cursus français pour la validation et l'adoption de nouvelles thérapeutiques, en particulier instrumentales, associées à un certain coût. S'il ne s'agissait que de décrire une fois de plus un paradoxe, cet éditorial pourrait paraître fade. Mais à l'image d'autres exemples déjà décrits, la situation actuelle connue par beaucoup peut être associée à une perte de chance pour le patient et illustre comment, sous des dogmes pseudoscientifiques, nous sommes capables de masquer une économie ou un défaut de moyen (ou de disposition à payer), alors que les soignés, soignants et décideurs (ou experts) au mieux s'interrogent ou au pire s'offusquent et accusent le manque de transparence des organismes de surveillance ou des traitements prescrits hors AMM.

La question du jour est celle du choix entre la surveillance endoscopique et le traitement par ablation endoscopique de la dysplasie de bas grade (DBG) prouvée et confirmée à deux reprises par deux pathologistes au cours de la surveillance de l'œsophage de Barrett (OB). Les recommandations actuelles et leurs révisions préconisent toujours une surveillance semestrielle de ces DBG jusqu'à régression (ou défaut d'échantillonnage !) ou évolution vers un stade plus évolué en dysplasie de haut grade (DHG) afin d'envisager un traitement endoscopique et/ou thermique, et pour certains encore, chirurgical!

Actuellement, nous disposons de deux études ouvertes prospectives, une multicentrique américaine de 2008 (Ganz et al., 2008) et une monocentrique néerlandaise de 2009 (Pouw et al., 2010) concernant le traitement par radiofréquence œsophagienne (RFO) de la DHG et la preuve (Shaheen et al., 2009) sous la forme d'une étude américaine prospective randomisée et contrôlée de l'efficacité de la RFO en cas d'OB et DBG ou par ailleurs OB et DHG sans

\footnotetext{
D. Heresbach $(\bowtie)$

Unité d'endoscopie digestive, centre hospitalier de Cannes,

15, avenue des Broussailles, CS 50008,

F-06414 Cannes, France

e-mail : d.heresbach@ch-cannes.fr
}

traitement de résection endoscopique préalable. Les résultats détaillés font l'objet d'un commentaire d'articles dans ce numéro.

Nous disposons, en outre d'une étude prospective multicentrique, du traitement du OB sans DBG ni DHG (Fleischer et al., 2010) avec un recul de cinq ans après la première séance de RFO. Pour ne citer que les résultats, avec le recul le plus important de cinq ans (soit 4,5 ans après la dernière séance à raison de 3,4 séances par patient), l'éradication de la métaplasie intestinale (MI) était de $92 \%$ en per protocole de $77 \%$ en intention de traiter et de respectivement 100 et $80 \%$ si la quatrième séance de RFO (au-delà des trois prévues au maximum) en cas de rémission incomplète ( $8 \%$ des patients) était prise en compte.

Certes, il faut analyser ces résultats aussi bien pour l'OB avec DBG ou DHG ou l'OB avec MI isolée en parallèle des effets secondaires et de la morbidité qui font l'objet d'un commentaire d'articles dans ce numéro, ainsi que de l'étude du ratio coût/efficacité, mais qui d'emblée ne constituent que des arguments supplémentaires pour dénoncer cette « viscosité décisionnelle ».

Nous avons déjà hérité de la pratique aéronautique des procédures d'évaluation des pratiques à risque et de la check-list, ce qui n'est pas un gros mot dans une médecine de plus en plus technologique. La pratique de simulation dans l'aéronautique montre que les pilotes entraînés avec des séances d'actualisation des connaissances (ou d'EPP ?) font face à quatre imprévus avec une réaction adaptée permettant de corriger la procédure et de mener le vol à bonne destination sans que les passagers n'aient eu connaissance des avaries et des conséquences potentiellement dramatiques.

Ainsi, 1, 2 et 3 montrent que nous sommes arrivés à trois avaries décisionnelles « imprévues » dont les « passagers ou cotisant $»$ n'ont pas connaissance ?

a) Le dépistage du cancer du côlon en population a autorisé 30 à $50 \%$ de faux-négatifs ;

b) ce dépistage indirect non seulement peu sensible n'a pas, plus que d'autres méthodes potentielles de dépistage, fait la 
preuve de la diminution de la mortalité spécifique ou globale en population à horizon de 25 ans chez les sujets âgés de 50 à 75 ans, atout et argument majeurs initiaux du choix scientifique (ou économique ?) pour le dépistage de masse ;

c) et enfin maintenant la poursuite de la surveillance des DBG sur OB sans les traiter.

Devrons-nous attendre, à l'instar d'un autre exemple médicamenteux et récent pour lequel l'ensemble de la société " soignées, soignantes et politique française » s'étonne a posteriori d'avoir ignoré la situation pendant
11 ans (1999-2010), pour s'offusquer une fois de plus que nous avons des instances et des acteurs ou décideurs visqueux ignorant ou n'ayant pas le courage d'annoncer la réalité des limites économiques auxquelles nous sommes soumis en 2011. À défaut, ou pour éviter l'aveu d'une médecine à deux vitesses, nous " gobons » une vérité à deux vitesses mais les responsabilités morales, voire les culpabilités, seront-elles à deux vitesses, ou rattraperont-elles à grande vitesse les attentistes visqueux ? Ces éléments nous invitent à nous interroger s'il y a un pilote dans l'avion et si oui, qui est-il ? 